\title{
Mitochondrial genome instability in colorectal adenoma and adenocarcinoma
}

Article in Tumor Biology · June 2015

DOI: 10.1007/s13277-015-3640-7 · Source: PubMed

CITATIONS

6

12 authors, including:

Aline Fonseca

10 PUBLICATIONS 88 CITATIONS

SEE PROFILE

\section{Julio Cesar Cetrulo Lorenz}

The Rockefeller University

36 PUBLICATIONS 420 CITATIONS

SEE PROFILE
READS

78

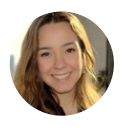

Jessica Plaça

University of São Paulo

16 PUBLICATIONS 16 CITATIONS

SEE PROFILE

Greice Andreotti Molfetta

University of São Paulo

33 PUBLICATIONS 329 CITATIONS

SEE PROFILE

Some of the authors of this publication are also working on these related projects:

Project Mental Deficiency Etiology in Brazilian Populations View project

Project $\quad$ Sickle Cell Disease and Stroke View project 


\title{
Mitochondrial genome instability in colorectal adenoma and adenocarcinoma
}

\author{
Luiza F. de Araujo ${ }^{1,2}$ • Aline S. Fonseca ${ }^{1,2}$ • Bruna R Muys ${ }^{1,2}$ • Jessica R. Plaça ${ }^{2}$. \\ Rafaela B. L. Bueno ${ }^{1,2}$ - Julio C. C. Lorenzi ${ }^{1,2}$ - Anemari R. D. Santos ${ }^{2}$ • \\ Greice A. Molfetta ${ }^{1,2,3}$ • Dalila L. Zanette ${ }^{1,2,3}$ - Jorge E. S. Souza ${ }^{2,3}$. \\ Valeria Valente $^{2,3,4}$ • Wilson A. Silva Jr $\mathbf{r}^{1,2,3}$
}

Received: 17 March 2015 / Accepted: 3 June 2015

(C) International Society of Oncology and BioMarkers (ISOBM) 2015

\begin{abstract}
Mitochondrial dysfunction is regarded as a hallmark of cancer progression. In the current study, we evaluated mitochondrial genome instability and copy number in colorectal cancer using Next Generation Sequencing approach and qPCR, respectively. The results revealed higher levels of heteroplasmy and depletion of the relative mtDNA copy number in colorectal adenocarcinoma. Adenocarcinoma samples also presented an increased number of mutations in nuclear genes encoding proteins which functions are related with mitochondria fusion, fission and localization. Moreover, we found a set of mitochondrial and nuclear genes, which cooperate in the same mitochondrial function simultaneously mutated in adenocarcinoma. In summary, these results support an important role for mitochondrial function and genomic instability in colorectal tumorigenesis.
\end{abstract}

Electronic supplementary material The online version of this article (doi:10.1007/s13277-015-3640-7) contains supplementary material, which is available to authorized users.

Wilson A. Silva, Jr

wilsonjr@usp.br

1 Department of Genetics, Ribeirão Preto Medical School, University of São Paulo, Ribeirão Preto, Brazil

2 Center for Cell-Based Therapy (CEPID/FAPESP); National institute of Science and Technology in Stem Cell and Cell Therapy (INCTC/ CNPq), Regional Blood Center of Ribeirão Preto, Riberão Preto, Brazil

3 Center for Medical Genomics (HCFMRP/USP), Center for Integrative Systems Biology (CISBi - NAP/USP), Ribeirão Preto, Brazil

4 Department of Clinical Analysis, Faculty of Pharmaceutical Science of Araraquara, University of São Paulo State, Araraquara, Brazil
Keywords Mitochondrial genome $\cdot$ Colorectal cancer . Heteroplasmy $\cdot$ Genome instability

\section{Introduction}

In worldwide cancer statistics, colorectal cancer is the third most common cancer diagnosed in men and second most common in women [1], while in Brazil, it is the fourth most common cancer in men and the third in women [2]. Colorectal cancer progress from multiple stages of adenomatous polyps, namely early adenoma, intermediate adenoma, and late adenoma, which are the precursor lesions of colorectal adenocarcinoma [3]. Fearon and Vogelstein [4] demonstrated that key genetic alterations are mutations in APC, KRAS, SMAD4/ CDC4, and TP53 that correlates with each stage of adenocarcinoma progression.

Additionally, Weinberg et al. [5] showed that proliferation was inhibited when cancer cells were treated with mitochondria-targeted antioxidants, demonstrating that mitochondrial ROS is required to stimulate cellular proliferation. The oxidative phosphorylation system (OXPHOS) is the main source of mitochondrial ROS [6]. Although it was previously known that OXPHOS is downregulated in cancer cells [7,8], some studies have suggested that OXPHOS is partially functional and required for carcinogenesis [9, 10]. OXPHOS is composed by four protein complexes embedded in the mitochondrial inner membrane, NADH dehydrogenase, succinate dehydrogenase, cytocrome c oxidoreductase, and cytocrome c oxidase [11]. All mitochondrial protein complexes, except for complex II, are constituted by proteins encoded in both mitochondrial and nuclear genome. The mitochondrial genome (mtDNA) is a circular molecule with 16,569 pair bases (pb) that contains 37 
genes: 22 transfer RNAs (tRNA), two ribosomal RNAs (rRNA), and 13 protein coding genes encoding members of the OXPHOS [12].

Mitochondrial genetic alterations can lead to OXPHOS dysfunction and play an important role in carcinogenesis [13]. In a study using cybrids (hybrid cytoplasm), Ishikawa and Hayashi [14] showed that low metastatic cells containing mitochondria derived from a high metastatic cell line displayed a high metastatic phenotype. [15], also using cybrids, showed that non-cancerous mitochondria inhibited oncogenic pathways, suggesting a mitochondrial-nucleus crosstalk in oncogenic regulation. Moreover, it was recently proposed that the complete lack of mtDNA is not beneficial for tumor growth and that some level of mitochondrial genomic instability can contribute to tumorigenesis [10].

Many studies have analyzed the mtDNA in colorectal cancer and demonstrated an increased instability in colorectal cancer using microsatellites and mtDNA copy number [16-19]. Here, we used next generation sequencing to analyze mitochondrial genome instability and genetic alterations in nuclear genes related with mitochondrial functions, and qPCR to evaluate mtDNA genome copy number. We observed higher levels of heteroplasmy, which is defined by the coexistence of different mtDNA in the same cell or tissue [20], and depletion of the relative mtDNA copy number in colorectal adenocarcinoma. More importantly, we found a set of mitochondrial and nuclear genes, involved in the same functions, simultaneously mutated in adenocarcinoma samples. Altogether, these data indicate that mitochondrial function and genomic instability have a fundamental role for colorectal cancer progression.

\section{Materials and methods}

\section{Tissue sample collections and ethics statement}

In this study, we analyzed nine patients (five men and four women) with clinical diagnosis of colorectal cancer submitted to surgical treatment at the Clinical Hospital of the Medical School of Ribeirao Preto of the University of São Paulo. From each patient, we collected normal tissue, adenoma, adenocarcinoma, and blood samples. The majority of patients presented age varying from 66 to 88 years old, except for one patient who was 35 years old at the diagnostic. According to histological criteria, one sample was classified as stage $\mathrm{T} 1$, one sample as T2, and six samples as T3, with tumors in ascending colon, sigmoid colon, and rectum (Table 1). All procedures were performed after approval of the Internal Human Ethics Committee (2374/2008 and 72902/2012) of the Clinical Hospital of the Medical School of Ribeirão Preto. Informed consent was obtained from all individual participants included in the study.
Adenoma and adenocarcinoma samples were collected from the same colonic topographic region (rectum, ascending, and sigmoid portions). Samples within the surgical safety border $(+5 \mathrm{~cm})$ were used as normal tissue. Histopathological examination confirmed that the adjacent tissues did not contain cancer cells. Peripheral blood was also collected from each patient. Tissue samples were stored in liquid nitrogen until analysis. DNA was extracted from tissue samples with TRIZOL $^{\circledR}$ (Life Technologies, US) and from blood samples with the Super Quik-Gene-Rapid DNA Isolation kit (Promega). Both techniques were performed according to manufacturers' guideline.

Inclusion criteria were as follows: all patients included should have both tissues, colorectal polyp (adenoma) and adenocarcinoma, confirmed by histopathological diagnosis. Exclusion criteria were as follows: patients diagnosed with Lynch syndrome, inflammatory bowel disease, or familial adenomatous polyposis (FAP), and patients that were previously submitted to chemotherapy and radiotherapy.

\section{mtDNA sequencing}

Ion Personal Genome Machine (PGM) was used for sequencing the mtDNA. Thus, three pairs of primers were designed for overlapped DNA regions covering the whole mitochondrial genome (7.500, 8.500, and 4.919 bp; Table S1). To amplify such long fragments, LongRange PCR kit was applied according to manufacturers' guideline (Qiagen Inc., Germantown, MD). Thermal cycling conditions were as follows: $3 \mathrm{~min}$ at $93{ }^{\circ} \mathrm{C}$, followed by 35 cycles of $15 \mathrm{~s}$ at $93{ }^{\circ} \mathrm{C}, 30 \mathrm{~s}$ at respective annealing temperature, an extension time of $1 \mathrm{~min}$ per $\mathrm{kb}$ at $68{ }^{\circ} \mathrm{C}$ (Table S1), and holding of $4{ }^{\circ} \mathrm{C}$.

Then, the Wizard SV Gel and PCR Clean-Up System kit (Promega) was used to purify amplicons. Quantifications were made with a QuantiFluor TM (Promega). Integrity of amplicons was checked with a DNA 12000 kit for Bioanalyzer 2100 System (Agilent).

Shear Plus kit was applied for enzymatic fragmentation of long amplificons. Ion Xpress Plus Fragment Library and Ion Xpress Barcode Adaptors kits (Life Technologies) were applied on chopped amplicons, for adaptors and barcodes ligation.

mtDNA fragments around $200 \mathrm{bp}$ were selected with E-Gel SizeSelect according to manufacturers' guidelines (Life Technologies). Agencourt AMPure XP kit (Beckman Coulter) was applied for purification of selected fragments. Libraries were then quantified with Ion Library Quantification kit (Life Technologies) in a 7500 Real-Time PCR system (Applied Biosystem).

Quantified libraries were used as template for Ion OneTouch $^{\mathrm{TM}} 200$ Template kit together with the Ion OneTouch $^{\mathrm{TM}}$ System (Life Technologies). This allowed the link single fragments to the Ion Sphere ${ }^{\mathrm{TM}}$ Particles (ISPs) 
Table 1 Clinical information of the patients

\begin{tabular}{lllllll}
\hline Patient & Gender & Age & Tumor site & Adenoma $^{\mathrm{a}}$ & Adenocarcinoma $^{\mathrm{b}}$ & Stage $^{\mathrm{c}}$ \\
\hline 1 & $\mathrm{M}$ & 77 & Sigmoid colon & T.A.L.D. & I.T.A & T1N0 \\
2 & $\mathrm{~F}$ & 88 & Rectum & T.V.A.L.D. & I.T.A & T2N2 \\
3 & $\mathrm{~F}$ & 69 & Rectum-sigmoid colon & T.A.L.D. & I.T.A & T3N1 \\
4 & $\mathrm{M}$ & 87 & Sigmoid colon & T.A.L.D. & I.T.A & T3N0 \\
5 & $\mathrm{~F}$ & 72 & Ascending colon & T.A.M.D. & I.T.A & T3N1 \\
6 & $\mathrm{M}$ & 66 & Rectum & T.A.M.D. & I.T.A & - \\
7 & $\mathrm{~F}$ & 35 & Sigmoid colon & T.A.M.D. & I.T.A & T3N1 \\
8 & $\mathrm{M}$ & 88 & Rectum & T.A.L.D. & I.T.A & T3N2 \\
9 & $\mathrm{M}$ & 75 & Rectum-sigmoid colon & T.A.L.H.D. & I.T.A & T3N2 \\
\hline
\end{tabular}

$M$ male, $F$ female

${ }^{a}$ T.A.L.D tubular adenoma with low grade dysplasia, T.A.M.D tubular adenoma with moderate dysplasia, T.A.L.H.D tubular adenoma with low and high-grade dysplasia, T.V.A.L.D tubulovillous adenoma with low grade dysplasia

${ }^{\mathrm{b}}$ I.T.A. invasive tubular adenocarcinoma

${ }^{\mathrm{c}}$ TNM Staging System (monoclonal sequences). Ion PGM 200 Sequencing kit (Life Technologies) and Ion 314 chip was used for sequencing.

In addition, we validated the mutations detected by Ion PGM sequencing using Sanger sequencing. First, we amplified the mtDNA fragments with primers and methods described in [21]. The sequencing was performed with BigDye Terminator v3.1 Cycle Sequencing kit in an ABI 3500XL Genetic Analyzer (Applied Biosystems).

\section{mtDNA sequencing analysis}

Ion PGM raw sequencing data were mapped against Revised Cambridge Reference Sequence (rCRS) (Andrews et al. 1999) with the TMAP aligner. Exclusion parameter for sequences were either polyclonal or Phred scores lower than $17(>=\mathrm{Q} 17)$. The identification and annotation of the variants were performed with the Variant Caller for the mtDNA plugin, specific for mtDNA variations, in the Ion Reporter Software. Geneious Basic 5.5.7 software was used to analyze the sequences obtained from the Sanger sequencing. The rate of heteroplasmy was calculated based on the ratio of the number of reads that mapped the mutated base to the number of reads that mapped the same nucleotide position. The MutPred and PolyPhen-2 softwares were used to predict the pathogenic effect of missense mutations $[22,23]$.

\section{mtDNA copy number analysis}

Two pairs of primers were designed using primer3 software $[24,25]$ (Table S2). One pair was used to amplify the mtDNA (Chr M: 9968-10150), while the other to target the nuclear DNA (Endogenous TUBB gene). Reactions with equal amounts of total DNA, as determined by NanoDrop spectrophotometer (Thermo Scientific, US), were performed with Power SYBR Green Master Mix (Life Technologies) in a 7500 Fast Real-Time System (Applied Biosystems). It determined the relative copy numbers of mitochondrial DNA compared to the nuclear DNA, as reported previously by Venegas et al. [26].

\section{Whole exome sequencing}

Genomic DNA was quantified using Qubit fluorometer (Life Technologies). Briefly, $50 \mathrm{ng}$ of DNA was used for library preparation with Nextera Exome Enrichment kit (Illumina Inc., San Diego, CA, USA). The probes in this kit were able to capture $62 \mathrm{Mb}$ of the exome content. The Exome DNA library obtained was quantified using the KAPA SYBR FAST (KAPA Biosystems), in the Fast Real-Time PCR System equipment (Life Technologies). The resulting purified DNA library was applied into an Illumina flow cell for cluster generation using the TruSeq PE Cluster Generation Kit v5 (Illumina Inc. San Diego, CA, USA) and sequenced on the Illumina Genome Analyzer IIx (GAIIx), with TruSeq SBS kit v5 (Illumina Inc), paired-end, $2 \times 76$ cycle run, following manufacturer's protocols.

Reads were aligned against the genome reference hg19 using BWA (v0.7.5a) [27]. Prior to variant calling, singletons were filtered out using samtools (v0.1.19) [28], .bam files were coordinated and sorted via Picard (v1.105, http:// picard.sourceforge.net/), and duplicates were removed via Picard. Prior to running GATK, read groups were added via Picard, and .bam file was re-ordered according to chromosome karyotype. Variants were called using the 
UnifiedGenotyper of the GATK (v.2.8.1) based upon established best practices [29].

For the analysis of exome data, we selected subsets of genes involved in different mitochondrial functions, namely mitochondrial complexes assembly, mitochondrial stability, protein synthesis, membrane polarization and potential (MPP), mitochondrial transport, small molecule transport (SMT), targeting protein to mitochondria (TPTM), mitochondrion protein import (MPI), electron transportation chain (ETC), outer membrane translocation (OMT), inner membrane translocation (INT), mitochondrial fission and fusion (FF), mitochondrial localization and apoptotic genes. As some genes are involved in multiple functions, they account for different gene subsets (Table S6).

\section{Statistical analysis}

Statistical analysis was performed using the GraphPad Prism Software (v.6.0, 2014). Analysis of Variance followed by Tukey's multiple comparisons tests was applied for the heteroplasmy and mtDNA copy number analysis and differences in number of mutations, affecting mitochondrial and nuclear genes subsets. A probability of $P<0.05$ was considered to be statistically significant.

\section{Results}

\section{Identification and characterization of mtDNA mutations}

A total of 24 mitochondrial genomes were successfully sequenced from the nine patients: eight adenoma and nine adenocarcinoma tissues, and seven blood samples. Three samples were excluded due to DNA low quality. Adenocarcinoma and blood samples from patient 1 (Table 1) were sequenced directly by the Sanger method. Other samples were sequenced by NGS in two runs: in the first run, adenocarcinoma and blood samples from patients 2 to 8 and in the second run, adenocarcinoma and blood samples from the patient 9 and adenoma samples from all patients. At the first run, we achieved an average coverage of $350 \times$ per genome; however, at the second run, we obtained an average depth of coverage of $23 \times$. In order to minimize errors, we only considered variants supported by at least five reads. Sequences have been deposited in GeneBank, and accession numbers can be found in supplementary material (Table S8). Tumor analysis revealed that 126 out of $224(56.3 \%)$ mutations occurred in protein coding genes, from which two $(0.9 \%)$ were indels, and $70(31.3 \%)$ and 54 (24.1\%) were synonymous and non-synonymous mutations, respectively. The other $98(43.8 \%)$ mutations were found in D-loop region, tRNA and rRNA genes (Table S3).

Then, we investigated if there was any preferentially mutated gene in blood, adenoma, or adenocarcinoma samples. For that analysis, we normalized the number of mutations by the total base-pair of each gene. We did not find any difference among samples; however, MT-ND6 gene was highly mutated in all tissues when compared to other genes (Table S4) (Fig. 2a). Similar results were observed when we considered only non-synonymous mutations (Fig. 2b) (Table S5).

\section{mtDNA somatic mutations}

We found that most of mutations $(75.9 \%, 170 / 224)$ were germinative, i.e., they were identified in all tissues (Fig 1b and Table S3). However, we also found that 27 mutations $(12.1 \%)$ were exclusive of adenocarcinoma and 11 (4.9\%) of adenoma samples, and two (1\%) were present in both tissues. Adenocarcinoma and adenoma somatic mutations are described in Tables 2 and 3, respectively.

Analysis of adenocarcinoma somatic mutations revealed that $11.1 \%(3 / 27)$ were found in the D-loop region, $14.8 \%$ $(4 / 27)$ in rRNA genes and $11.1 \%(3 / 27)$ in the tRNA genes. Higher mutation rates were found in complex I genes, with $37.0 \%$ (10/27), followed by complex IV, with $14.8 \%(4 / 27)$, and III, with $11.1 \%(3 / 27)$. Moreover in D-loop region, two mutations were found in the D310 sequence: $\mathrm{m} .309 \mathrm{C}_{5} \mathrm{~T}_{1}$ in adenocarcinoma samples and m.308 $\mathrm{C}_{5} \mathrm{~T}_{1}$ found both in adenomas and adenocarcinomas. In adenomas, $27.3 \%$ (3/11) of all the somatic mutations were found in the D-loop region and $9.1 \%(1 / 11)$ in rRNA genes. Complexes I and III showed a similar incidence of mutations, $27.3 \%$ (3/11). A lower frequency of mutation was observed in the complex V, $9.1 \%$ $(1 / 11)$.

Adenoma and adenocarcinoma samples showed the same proportion of somatic non-synonymous mutations (45.5\%, $5 / 11$ and $44.5 \%, 12 / 27$, respectively). However, different complexes were more affected by these mutations in each tissue. Non-synonymous mutations were more frequent in complex I genes $(66.7 \%, 8 / 12)$ (Fig. 2c) of adenocarcinoma samples and in the complex III genes of adenoma samples $(60.0 \%, 3 / 5)$ (Fig. 2d).

\section{Heteroplasmy analysis}

The heteroplasmy level is the ratio of distinct mitochondrial genomes in a cell or individual, and it can be precisely calculated using next-generation sequencing [13]. In our study, we identified which mutations were in heteroplasmy (heteroplasmic sites) and calculated their levels to infer the mtDNA genomic instability rate in colorectal cancer. Heteroplasmic sites represented 
Fig. 1 Heteroplasmy analysis in blood, adenoma, and adenocarcinoma samples. a Heteroplasmy levels were higher in colorectal adenocarcinoma when compared with blood mutations $(p=0.0329)$. b Venn diagram showing mtDNA mutations found the samples
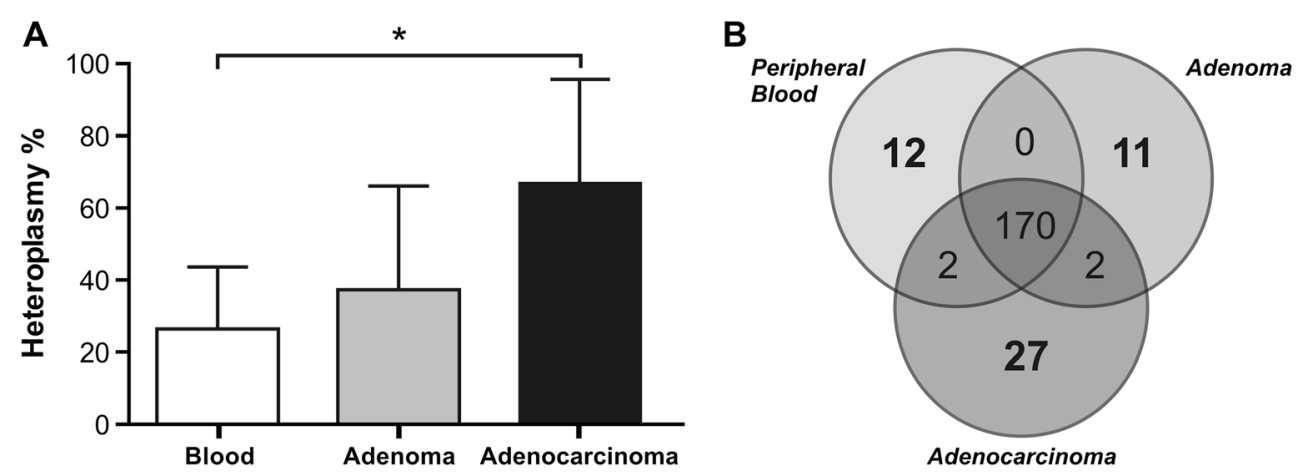

$41.6 \%(5 / 12), 45 \%(5 / 11)$, and $37 \%(10 / 27)$ of the somatic mutations identified in blood, adenoma, and adenocarcinoma samples, respectively. Although heteroplasmic sites were less represented in adenocarcinoma, their levels were higher when compared to blood samples $(p=0.0329)$ (Fig. 1a).

Table 2 Somatic mutations identified in colorectal adenocarcinoma

\begin{tabular}{|c|c|c|c|c|c|}
\hline Gene & Nucleotide change & Amino acid change & Heteroplasmy & PolyPhen score & MutPred score \\
\hline D-loop & $\mathrm{m} .308 \operatorname{ins}_{5} \mathrm{~T}_{1}^{\mathrm{a}, \mathrm{b}}$ & - & $\mathrm{HM}$ & - & - \\
\hline D-loop & m.309ins $\mathrm{C}_{5} \mathrm{~T}_{1}{ }^{\mathrm{b}}$ & - & $\mathrm{HM}$ & - & - \\
\hline D-loop & $\mathrm{m} .499 \mathrm{G}>\mathrm{A}^{\mathrm{b}}$ & - & $\mathrm{HM}$ & - & - \\
\hline MT-RNR2 & $\mathrm{m} .2065 \mathrm{~A}>\mathrm{G}$ & - & $\mathrm{HM}$ & - & - \\
\hline$M T-R N R 2$ & $m .2128 G>A$ & - & 18.87 & - & - \\
\hline$M T-R N R 2$ & $m .2571 G>A$ & - & 74.59 & - & - \\
\hline MT-RNR2 & $\mathrm{m} .2789 \mathrm{C}>\mathrm{T}$ & - & 65.64 & - & - \\
\hline MT-ND1 & $\mathrm{m} .3594 \mathrm{C}>\mathrm{T}^{\mathrm{b}}$ & V96V & $\mathrm{HM}$ & - & - \\
\hline$M T-t R N A$-Ile & m.4314delA & - & 81.43 & - & - \\
\hline MT-ND2 & $\mathrm{m} .4831 \mathrm{G}>\mathrm{A}^{\mathrm{b}}$ & G121D & HM & 1 & 0.797 \\
\hline MT-ND2 & $\mathrm{m} .4868 \mathrm{~A}>\mathrm{G}$ & W133W & 83.58 & - & - \\
\hline MT-tRNA Ala & $m .5669 G>A$ & - & $H M$ & - & - \\
\hline$M T-C O I$ & m.6816ins $C$ & - & $H M$ & - & - \\
\hline MT-COI & $\mathrm{m} .6817 \mathrm{~T}>\mathrm{C}$ & F305S & $\mathrm{HM}$ & 0.999 & 0.696 \\
\hline MT-COI & $\mathrm{m} .7256 \mathrm{C}>\mathrm{T}^{\mathrm{b}}$ & $\mathrm{N} 451 \mathrm{~N}$ & HM & - & - \\
\hline MT-COII & $m .7910 G>A$ & $E 109 K$ & 91.89 & 1 & 0.756 \\
\hline MT-COIII & $m .9545 \mathrm{del} G^{\mathrm{a}}$ & - & 74.71 & - & - \\
\hline MT-tRNA Arg & m.10406G $>$ A & - & 44.3 & - & - \\
\hline$M T-N D 4 L$ & $m .10570 A>T$ & $E 34 K$ & 20.63 & 0.131 & 0.825 \\
\hline MT-ND4 & $m .11651 G>A$ & V298M & $H M$ & 0.85 & 0.48 \\
\hline MT-ND5 & $m .12667 G>A$ & $D 111 N$ & 75.00 & 0.999 & 0.841 \\
\hline MT-ND5 & $\mathrm{m} .12773 \mathrm{G}>\mathrm{A}$ & G146D & $\mathrm{HM}$ & 1 & 0.299 \\
\hline MT-ND5 & $\mathrm{m} .13042 \mathrm{G}>\mathrm{A}$ & $\mathrm{A} 236 \mathrm{~T}$ & $\mathrm{HM}$ & 0.999 & 0.748 \\
\hline MT-ND5 & $\mathrm{m} .13153 \mathrm{~A}>\mathrm{G}$ & $\mathrm{I} 273 \mathrm{~V}$ & 92.41 & 0.003 & 0.484 \\
\hline MT-ND6 & $\mathrm{m} .14318 \mathrm{~T}>\mathrm{C}$ & N119S & $\mathrm{HM}$ & 0.013 & 0.344 \\
\hline MT-CYB & $\mathrm{m} .14971 \mathrm{~T}>\mathrm{C}$ & $\mathrm{Y} 75 \mathrm{Y}$ & 93.53 & - & - \\
\hline$M T-C Y B$ & $m .15276 G>A$ & $R 177 Q$ & $H M$ & 1 & 0.833 \\
\hline MT-CYB & $\mathrm{m} \cdot 15843 \mathrm{~T}>\mathrm{C}^{\mathrm{a}}$ & M366T & HM & 0.238 & 0.433 \\
\hline D-loop & m.16153G $>A^{b}$ & - & $\mathrm{HM}$ & - & - \\
\hline
\end{tabular}

The mutations in italic have not been reported in the Mitomap database [40]

${ }^{\text {a }}$ Common mutation in colorectal adenoma and adenocarcinoma

${ }^{\mathrm{b}}$ Reported in other cancer studies

$H M$ homoplasmic mutation 
Table 3 Somatic mutations identified in colorectal adenomas

\begin{tabular}{lllcll}
\hline Gene & Nucleotide change & Amino acid change & Ploidy & PolyPhen score & MutPred score \\
\hline D-loop & m.66G $>\mathrm{T}$ & - & 52.0 & - & - \\
D-loop & m.308insC $_{5} \mathrm{~T}_{1}^{\mathrm{a}, \mathrm{b}}$ & - & $\mathrm{HM}$ & - & - \\
D-loop & m.567insC & - & $\mathrm{HM}$ & - & - \\
MT-RNR1 & m.827A $>\mathrm{G}$ & - & $\mathrm{HM}$ & - & - \\
MT-COIII & m.9545delG & - & 72.3 & - & - \\
MT-ND2 & m.4977T $>\mathrm{C}^{\mathrm{b}}$ & $\mathrm{L} 170 \mathrm{~L}$ & $\mathrm{HM}$ & - & - \\
MT-ATP6 & $\mathrm{m} .8911 \mathrm{~T}>\mathrm{C}$ & $\mathrm{L} 129 \mathrm{~L}$ & 4.39 & - & - \\
MT-ND4 & $\mathrm{m} .11435 \mathrm{G}>\mathrm{A}$ & $\mathrm{A} 676 \mathrm{~T}$ & $\mathrm{HM}$ & 0.999 & 0.743 \\
MT-ND5 & $\mathrm{m} .13597 \mathrm{G}>\mathrm{A}$ & $\mathrm{G} 1261 \mathrm{~A}$ & $\mathrm{HM}$ & 0.999 & 0.431 \\
MT-CYB & $\mathrm{m} .15062 \mathrm{~T}>\mathrm{C}$ & $\mathrm{S} 316 \mathrm{P}$ & 8.08 & 0.999 & 0.855 \\
MT-CYB & $\mathrm{m} .15282 \mathrm{~T}>\mathrm{C}$ & $\mathrm{F} 536 \mathrm{~S}$ & 59.68 & 1 & 0.793 \\
MT-CYB & $\mathrm{m} .15762 \mathrm{G}>\mathrm{A}$ & $\mathrm{G} 1016 \mathrm{E}$ & 61.11 & 1 & 0.877 \\
D-loop & $\mathrm{m} .16468 \mathrm{~T}>\mathrm{C}$ & - & $\mathrm{HM}$ & - & - \\
\hline
\end{tabular}

The mutations in italic have not been reported in the Mitomap database [40]

${ }^{a}$ Common mutation in colorectal adenoma and adenocarcinoma

${ }^{\mathrm{b}}$ Reported in other cancer studies

$H M$ homoplasmic mutation

\section{Mutational cross-analysis of mitochondrial and nuclear genes}

Additionally, we performed whole exome sequencing of normal colon, adenoma, and adenocarcinoma samples of patients 2,3 , and 4 (Table 1). Due to the small number of mutations detected in adenoma tissues, in order to identify any nuclear gene subset preferentially mutated in adenocarcinoma samples, we considered only mutations found in adenocarcinoma and normal colon samples. We also normalized the number of mutations per gene, once the number of genes in each subset varied (Table S6). Among all analyzed categories, we found
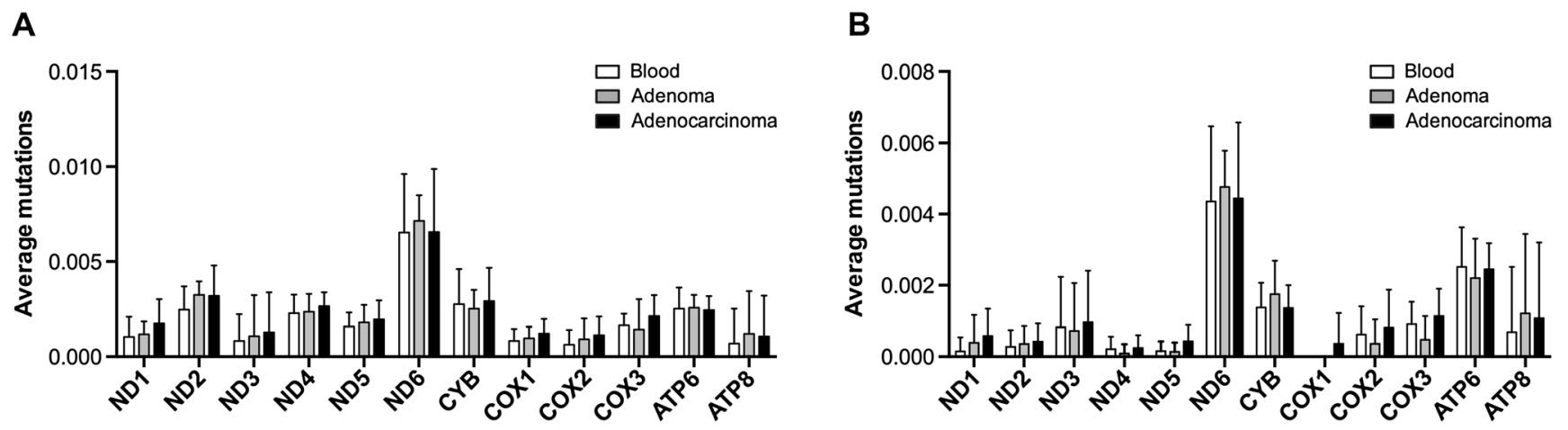

C

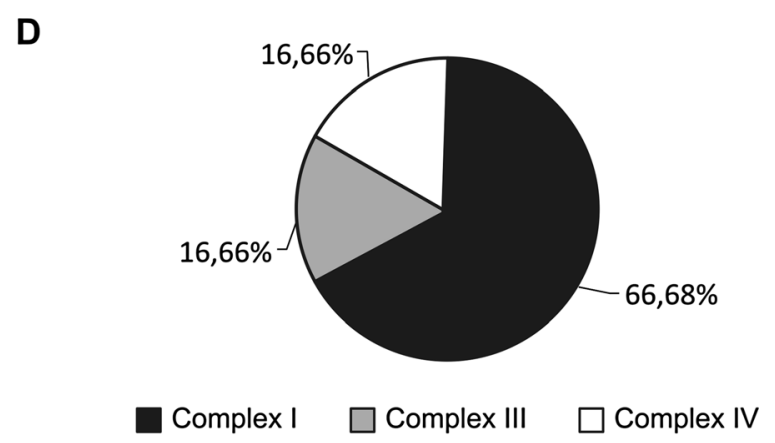

Fig. 2 mtDNA mutations frequencies per gene. a Total average of mutations and $\mathbf{b}$ average non-synonymous mutations per gene showed that MT-ND6 gene was highly affected in all tissues (Table S4 and S5). c,

d Represent the percentage of non-synonymous somatic mutations that affected the mitochondrial complexes found in adenoma samples (c) and adenocarcinoma samples (d) 
that only fusion/fission $(p=0.0472)$ and mitochondrial localization $(p=0.0231)$ genes were more affected in adenocarcinoma than in normal colon (Fig. 3a).

Mutations identified in adenocarcinoma samples are listed in Table S7. The most affected gene in adenocarcinoma was SLC25A5 with 11 mutations, followed by POLG and COX10, both with five mutations (Table S7). We also observed that several genes involved in the same mitochondrial function were simultaneously mutated in nuclear and mitochondrial genomes, namely mitochondrial complex I genes NDUFS3, NDUFS4, NDUFS5, NDUFS6, NDUFB10, NDUFA7, NDUFA10, MT-ND1, MT-ND2, and MT-ND6, and genes related to mitochondrial translation TSFM, GFM1, and MT-tRNAs (red lines in Fig. 4).

Additionally, we evaluated the changes in mtDNA copy number in normal colon, adenoma, and adenocarcinoma samples. We observed a decrease of mtDNA copies in adenocarcinoma compared to normal colon $(p=0.0092)$ (Fig. 3b).

\section{Discussion}

Several studies have analyzed mitochondrial instability comparing normal and colorectal tumor tissues [17-19]. However, only a few have reported mtDNA instability in different stages of colorectal cancer [18, 19]. In our study, we demonstrated higher levels of mtDNA instability, and that nuclear and mitochondrial genes involved in the same function are simultaneously mutated in colorectal adenocarcinoma. These results suggest that genomic instability of genes related with mitochondrial function can impact tumor progression, corroborating previous hypothesis of the existence of mitochondrial-nuclear cross-talk in the regulation of oncogenic pathways [15] and that mtDNA instability can act as a cancer hallmark [30].

\section{Somatic mtDNA mutation in colorectal cancer}

There is evidence that somatic mutations in mtDNA might play a role in cancer development $[31,14,32]$. Polyak et al. [33] published the first paper describing somatic mtDNA mutations in colorectal cancer. Here, we identified novel mtDNA mutations exclusive of colorectal adenoma or adenocarcinoma (Fig. 1b, Tables 2 and 3). We have also found mutations that were previously described in cancer and other diseases. For instance, the mutation $\mathrm{m} .13042 \mathrm{G}>\mathrm{A}$ (Ala236Thr) in the ND5 gene was reported in patients diagnosed with mitochondrial disease and severe complex I impairment [34, 35], and the mutation $\mathrm{m} .4831 \mathrm{G}>\mathrm{A}$ (Gly121Asp) in the ND2 gene was associated with anchorage-independent proliferation of cancer cell lines, increased ROS production, HIF $1 \alpha$ stabilization, and glucose metabolism alteration [36, 37].

A recent study showed that mitochondrial heteroplasmatic sites are commonly present in healthy individuals [13]. However, increased levels of heteroplasmy are associated with the tumorigenesis [16] and can predict if mutant phenotypes will be expressed [38]. Here, we found similar frequencies of heteroplasmic sites among analyzed tissues and higher levels of heteroplasmy in colorectal adenocarcinoma $(p=0.0329)$ (Fig. 1a). Some studies suggest that heteroplasmic mutations associated with mitochondrial dysfunction might confer a selective advantage for oncogenesis [16, 17, 38].

\section{Cross-talk of mtDNA and nuclear gene mutations}

Here, we demonstrated that several genes of the complex I were highly mutated in both genomes (Fig. 4), with MTND6 being the most instable gene (Fig. 2a, b). Recently, it has been reported that the complete lack of mtDNA delays tumor progression, suggesting that some level of OXPHOS is required for tumorigenesis and metastasis [10]. Many cancer studies have reported that complex I genes are preferentially
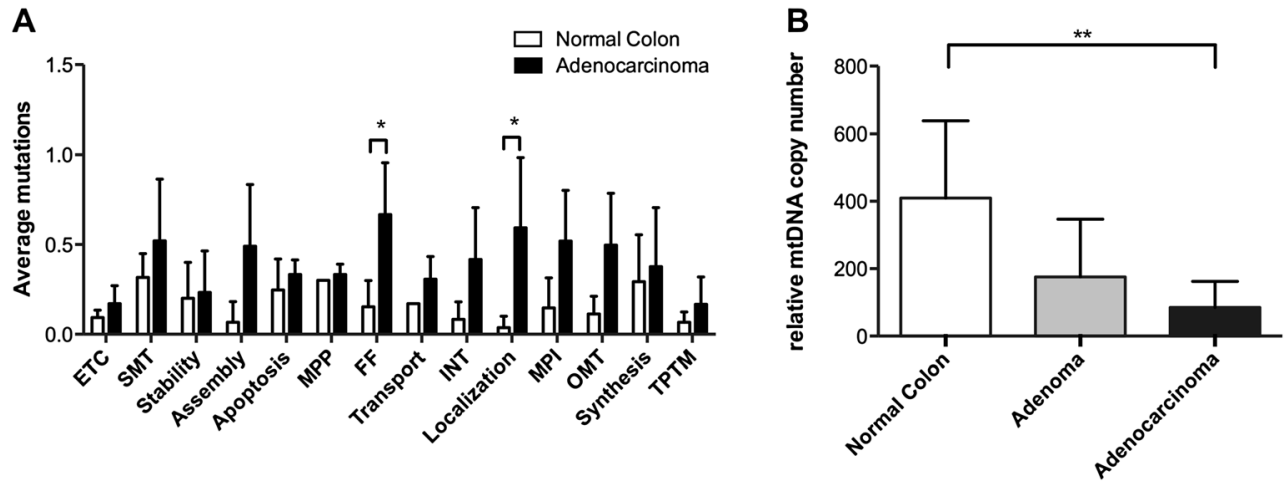

Fig. 3 Mutations frequencies per nuclear genes subset and mtDNA copy number analysis. a Average adenocarcinoma and normal-colon mutations found in each subset of nuclear genes related with mitochondrial functions. Statistical analysis revealed that fusion and fission $(p=$
$0.0472)$ and mitochondrial localization genes subsets $(p=0.0231)$ were more affected in adenocarcinoma samples. b Relative mtDNA copy number analysis showed a decreased number of mitochondrial genome content in adenocarcinoma when compared to normal colon $(p=0.0092)$ 


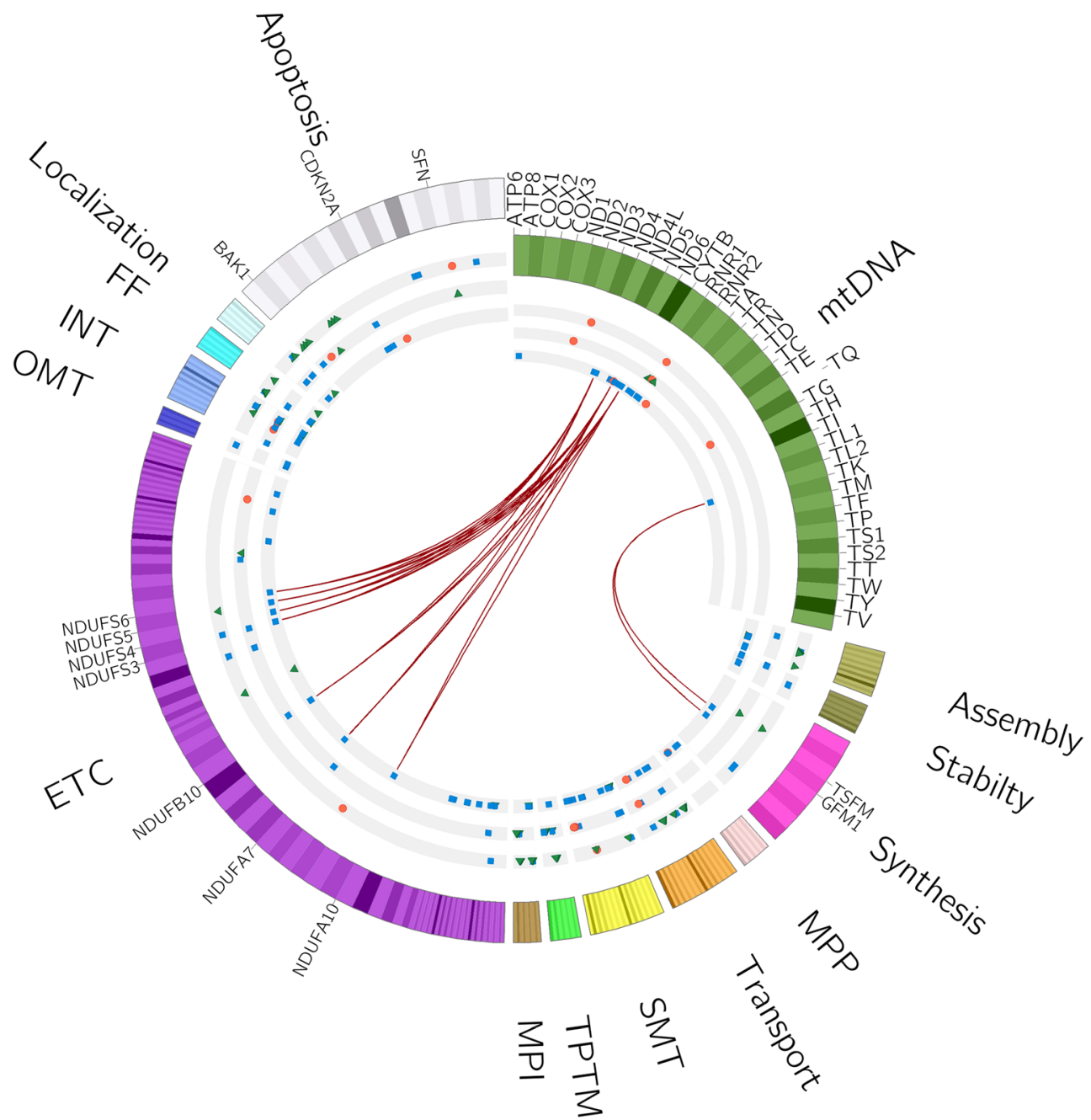

Fig. 4 Circos plot representing the mitochondrial genome (mtDNA) and each subset of nuclear genes involved in mitochondrial functions: mitochondrial complexes assembly (Assembly), mitochondrial stability (stability), protein synthesis (synthesis), membrane polarization and potential (MPP), mitochondrial transport (transport), small molecule transport (SMT), targeting protein to mitochondria (TPTM), mitochondrion protein import (MPI), electron transportation chain (ETC), outer membrane translocation (OMT), inner membrane translocation (INT), mitochondrial fission and fusion (FF),

mutated in tumors [39, 32], and those mutations were associated with cancer progression mediated by ROS production, $\mathrm{PI} 3 \mathrm{~K} / \mathrm{Akt} / \mathrm{PKC}$ pathway activation, and $\operatorname{HIF} 1 \alpha[14,13,31$, 40].

Interestingly, we also found mutations in mitochondrial and nuclear genes involved in mitochondrial translation, such as m.4314delA, m.5669G $>$ A, m.10406G $>$ A, rs77102248, rs7187776, and rs3088215 (Fig. 4, Table S3 and S7). Mutations in tRNA genes can modify their secondary structure in mitochondria [41], interfering in the RNA stability and in the translational machinery [42]. Also, mutations in elongation factors EFG1 and EFTS were associated with decreased levels of oxidative phosphorylation complexes, defects in mitochondrial localization (localization), and apoptotic genes (apoptosis). Patients 2 (inner), 3 (intermediate), and 4 (outer) are represented in the layers. For each patient, germinative mutations (blue square), adenocarcinoma mutations (red circle), and mutations present in both adenoma and adenocarcinoma (green triangle) mutations are represented within each gene subset. Red lines connect mutated genes from the nuclear and mitochondrial genome that act in the same pathway. Circos plot was draw with Circos.ca [53]

mitochondrial translation [43], and reduced amount of assembled complexes I, IV, and V [44].

Moreover, we demonstrated that FF and mitochondrial localization genes were more affected in adenocarcinoma when compared to normal colon. Mutations in localization, and fusion and fission genes can affect the cell dynamics. Desai et al. [45] showed that mitochondrion redistribution to the anterior region of the cancer cell is required for persistent migration. Also, a study with breast cancer cell lines showed that expression of FF genes regulates cell migration and invasion [46].

It was previously hypothesized that nuclear and mtDNA mutations are co-selected to promote cancer cell survival [7]. Our study demonstrated that some nuclear 
and mitochondrial genes that cooperate in the same mitochondrial function were mutated in adenocarcinoma samples. We further suggest that mutations in both genomes are necessary to promote the mitochondrial impairment required for tumorigenesis. Functional studies are necessary to confirm this hypothesis.

\section{mtDNA copy number analysis}

mtDNA copy number variation have also been associated with human cancer progression [47]. Those alterations can result in the loss of mitochondrial transmembrane potential and induce a mitochondrial retrograde signaling, resulting in altered expression of nuclear genes [48]. Our results showed a decreased mtDNA copy number in adenocarcinoma (Fig. 3b), which are in accordance with previous studies $[49,50]$. The mtDNA depletion could be explained by mutations in D-loop region and POLG gene, both involved with mtDNA synthesis $[49,51]$, and by mutations in fusion and fission genes that were previously associated with mtDNA depletion [52].

\section{Conclusion}

The present study reports for the first time the analysis of mitochondrial genome instability in noncancerous tissues, adenoma, and adenocarcinoma from the same patients. The results demonstrated increased heteroplasmy level and mtDNA depletion, features that can lead to mitochondrial dysfunction in colorectal cancer. Moreover, we found an enrichment of mutations in genes involved with fusion and fission, and mitochondrial localization, suggesting an alteration in the mitochondrial dynamics in adenocarcinoma. Our data also indicates that nuclear and mitochondrial mutations cooperate to enhance the mitochondrial impairment in cancer cells. Further studies are necessary to functionally characterize the impact of this impairment in cancer development.

Acknowledgments We thank Adriana Aparecida Marques and Life Technologies (Brazil) for the technical support. We also thank Vinicius Kannen Cardoso for the scientific insights.

Ethical approval All procedures performed in studies involving human participants were in accordance with the ethical standards of the institutional and/or national research committee and with the $1964 \mathrm{Hel}-$ sinki declaration and its later amendments or comparable ethical standards.

Funding This work was funding by The National Council for Scientific and Technological Development (CNPq), grant \#573754/2008-0; by grants \#2008/57877-3 and \#2013/08135-2, São Paulo Research Foundation (FAPESP); and by Research Support of the University Sao Paulo, CISBi-NAP/USP \#12.1.25441.01.2.

Conflicts of interest None.

\section{References}

1. Jemal A, Bray F, Center MM, Ferlay J, Ward E, Forman D. Global cancer statistics. CA Cancer J Clin. 2011;61(2):69-90. doi:10.3322/ caac.20107.

2. INCA. Estimativa 2014-Incidência de Câncer no Brasil. Rio de Janeiro, RJ. 2014. Accessed 04/28/15.

3. Cunningham D, Atkin W, Lenz HJ, Lynch HT, Minsky B, Nordlinger B. Colorectal cancer. Lancet. 2010;375(9719):1030 47. doi:10.1016/S0140-6736(10)60353-4.

4. Fearon ER, Vogelstein B. A genetic model for colorectal tumorigenesis. Cell. 1990;61(5):759-67.

5. Weinberg F, Hamanaka R, Wheaton WW, Weinberg S, Joseph J, Lopez M, et al. Mitochondrial metabolism and ROS generation are essential for Kras-mediated tumorigenicity. Proc Natl Acad Sci U S A. 2010;107(19):8788-93. doi:10.1073/pnas.1003428107.

6. Giannoni E, Buricchi F, Raugei G, Ramponi G, Chiarugi P. Intracellular reactive oxygen species activate Src tyrosine kinase during cell adhesion and anchorage-dependent cell growth. Mol Cell Biol. 2005;25(15):6391-403. doi:10.1128/MCB.25.15.63916403.2005.

7. Gaude E, Frezza C. Defects in mitochondrial metabolism and cancer. Cancer Metab. 2014;2:10. doi:10.1186/2049-3002-2-10.

8. Warburg O. On the origin of cancer cells. Science. 1956;123(3191): 309-14.

9. Fantin VR, St-Pierre J, Leder P. Attenuation of LDH-A expression uncovers a link between glycolysis, mitochondrial physiology, and tumor maintenance. Cancer Cell. 2006;9(6):425-34. doi:10.1016/j. ccr.2006.04.023.

10. Tan AS, Baty JW, Dong LF, Bezawork-Geleta A, Endaya B, Goodwin J, et al. Mitochondrial genome acquisition restores respiratory function and tumorigenic potential of cancer cells without mitochondrial DNA. Cell Metab. 2015;21(1):81-94. doi:10.1016/j. cmet.2014.12.003.

11. Wallace DC. Structure and evolution of organelle genomes. Microbiol Rev. 1982;46(2):208-40.

12. Anderson S, Bankier AT, Barrell BG, de Bruijn MH, Coulson AR, Drouin J, et al. Sequence and organization of the human mitochondrial genome. Nature. 1981;290(5806):457-65.

13. Singh RK, Srivastava A, Kalaiarasan P, Manvati S, Chopra R, Bamezai RN. mtDNA germ line variation mediated ROS generates retrograde signaling and induces pro-cancerous metabolic features. Sci Rep. 2014;4:6571. doi:10.1038/srep06571.

14. Ishikawa K, Hayashi J. A novel function of mtDNA: its involvement in metastasis. Ann N Y Acad Sci. 2010;1201:40-3. doi:10. 1111/j.1749-6632.2010.05616.x.

15. Kaipparettu BA, Ma Y, Park JH, Lee TL, Zhang Y, Yotnda P, et al. Crosstalk from non-cancerous mitochondria can inhibit tumor properties of metastatic cells by suppressing oncogenic pathways. PLoS One. 2013;8(5):e61747. doi:10.1371/journal.pone.0061747.

16. He Y, Wu J, Dressman DC, Iacobuzio-Donahue C, Markowitz SD, Velculescu VE, et al. Heteroplasmic mitochondrial DNA mutations in normal and tumour cells. Nature. 2010;464(7288):610-4. doi:10. 1038/nature08802.

17. Larman TC, DePalma SR, Hadjipanayis AG, Protopopov A, Zhang J, Gabriel SB, et al. Spectrum of somatic mitochondrial mutations in five cancers. Proc Natl Acad Sci U S A. 2012;109(35):14087-91. doi:10.1073/pnas.1211502109.

18. Lee JH, Hwang I, Kang YN, Choi IJ, Kim DK. Genetic characteristics of mitochondrial DNA was associated with colorectal carcinogenesis and its prognosis. PLoS One. 2015;10(3):e0118612. doi: 10.1371/journal.pone.0118612.

19. Lim SW, Kim HR, Kim HY, Huh JW, Kim YJ, Shin JH, et al. Highfrequency minisatellite instability of the mitochondrial genome in 
colorectal cancer tissue associated with clinicopathological values. Int J Cancer. 2012;131(6):1332-41. doi:10.1002/ijc.27375.

20. Ye K, Lu J, Ma F, Keinan A, Gu Z. Extensive pathogenicity of mitochondrial heteroplasmy in healthy human individuals. Proc Natl Acad Sci U S A. 2014;111(29):10654-9. doi:10.1073/pnas. 1403521111

21. Taylor RW, Taylor GA, Durham SE, Turnbull DM. The determination of complete human mitochondrial DNA sequences in single cells: implications for the study of somatic mitochondrial DNA point mutations. Nucl Acids Res. 2001;29(15):E74-4.

22. Adzhubei IA, Schmidt S, Peshkin L, Ramensky VE, Gerasimova A, Bork P, et al. A method and server for predicting damaging missense mutations. Nat Methods. 2010;7(4):248-9. doi:10.1038/ nmeth0410-248.

23. Li B, Krishnan VG, Mort ME, Xin F, Kamati KK, Cooper DN, et al. Automated inference of molecular mechanisms of disease from amino acid substitutions. Bioinformatics. 2009;25(21):2744-50. doi:10.1093/bioinformatics/btp528.

24. Untergasser A, Cutcutache I, Koressaar T, Ye J, Remme M. Primer3-new capabilities and interfaces. Nucleic Acids Res. 2012;40(15):e115. doi:10.1093/nar/gks596.

25. Koressaar T, Remm M. Enhancements and modifications of primer design program Primer3. Bioinformatics. 2007;23(10):1289-91. doi:10.1093/bioinformatics/btm091.

26. Venegas V, Wang J, Dimmock D, Wong LJ. Real-time quantitative PCR analysis of mitochondrial DNA content. Current protocols in human genetics / editorial board, Jonathan L Haines [et al]. 2011; Chapter 19:Unit 19 7. doi:10.1002/0471142905.hg1907s68.

27. Li H, Durbin R. Fast and accurate short read alignment with Burrows-Wheeler transform. Bioinformatics. 2009;25(14):175460. doi:10.1093/bioinformatics/btp324.

28. Li H, Handsaker B, Wysoker A, Fennell T, Ruan J, Homer N, et al. The sequence alignment/map format and SAMtools. Bioinformatics. 2009;25(16):2078-9. doi:10.1093/bioinformatics/ btp352.

29. DePristo MA, Banks E, Poplin R, Garimella KV, Maguire JR, Hartl $\mathrm{C}$, et al. A framework for variation discovery and genotyping using next-generation DNA sequencing data. Nat Genet. 2011;43(5): 491-8. doi:10.1038/ng.806.

30. Gasparre G, Porcelli AM, Lenaz G, Romeo G. Relevance of mitochondrial genetics and metabolism in cancer development. Cold Spring Harb Perspect Biol. 2013;5(2). doi:10.1101/cshperspect. a011411.

31. Iommarini L, Kurelac I, Capristo M, Calvaruso MA, Giorgio V, Bergamini C, et al. Different mtDNA mutations modify tumor progression in dependence of the degree of respiratory complex I impairment. Hum Mol Genet. 2014;23(6):1453-66. doi:10.1093/ hmg/ddt533.

32. Tan AS, Baty JW, Berridge MV. The role of mitochondrial electron transport in tumorigenesis and metastasis. Biochim Biophys Acta. 2014;1840(4):1454-63. doi:10.1016/j.bbagen.2013.10.016.

33. Polyak K, Li Y, Zhu H, Lengauer C, Willson JK, Markowitz SD, et al. Somatic mutations of the mitochondrial genome in human colorectal tumours. Nat Genet. 1998;20(3):291-3. doi:10.1038/ 3108 .

34. Blok MJ, Spruijt L, de Coo IF, Schoonderwoerd K, Hendrickx A, Smeets HJ. Mutations in the ND5 subunit of complex I of the mitochondrial DNA are a frequent cause of oxidative phosphorylation disease. J Med Genet. 2007;44(4):e74. doi:10.1136/jmg.2006. 045716.

35. Naini AB, Lu J, Kaufmann P, Bernstein RA, Mancuso M, Bonilla E, et al. Novel mitochondrial DNA ND5 mutation in a patient with clinical features of MELAS and MERRF. Arch Neurol. 2005;62(3):473-6. doi:10.1001/archneur.62.3. 473.
36. Danovi D, Cremona CA, Machado-da-Silva G, Basu S, Noon LA, Parrinello S, et al. A genetic screen for anchorage-independent proliferation in mammalian cells identifies a membrane-bound neuregulin. PLoS One. 2010;5(7):e11774. doi:10.1371/journal. pone. 0011774 .

37. Zhou S, Kachhap S, Sun W, Wu G, Chuang A, Poeta L, et al. Frequency and phenotypic implications of mitochondrial DNA mutations in human squamous cell cancers of the head and neck. Proc Natl Acad Sci U S A. 2007;104(18):7540-5. doi:10.1073/pnas. 0610818104.

38. Zhang C, Huang VH, Simon M, Sharma LK, Fan W, Haas R, et al. Heteroplasmic mutations of the mitochondrial genome cause paradoxical effects on mitochondrial functions. FASEB J. 2012;26(12): 4914-24. doi:10.1096/fj.12-206532.

39. Porcelli AM, Ghelli A, Ceccarelli C, Lang M, Cenacchi G, Capristo $\mathrm{M}$, et al. The genetic and metabolic signature of oncocytic transformation implicates HIF1alpha destabilization. Hum Mol Genet. 2010;19(6):1019-32. doi:10.1093/hmg/ddp566.

40. Koshikawa N, Hayashi J, Nakagawara A, Takenaga K. Reactive oxygen species-generating mitochondrial DNA mutation upregulates hypoxia-inducible factor-1alpha gene transcription via phosphatidylinositol 3-kinase-Akt/protein kinase C/histone deacetylase pathway. J Biol Chem. 2009;284(48):33185-94. doi: 10.1074/jbc.M109.054221.

41. Kurelac I, MacKay A, Lambros MB, Di Cesare E, Cenacchi G, Ceccarelli C. Somatic complex I disruptive mitochondrial DNA mutations are modifiers of tumorigenesis that correlate with low genomic instability in pituitary adenomas. Hum Mol Genet. 2013;22(2):226-38. doi:10.1093/hmg/dds422.

42. Levinger L, Morl M, Florentz C. Mitochondrial tRNA 3' end metabolism and human disease. Nucleic Acids Res. 2004;32(18): 5430-41. doi:10.1093/nar/gkh884.

43. Coenen MJ, Antonicka H, Ugalde C, Sasarman F, Rossi R, Heister $\mathrm{JG}$, et al. Mutant mitochondrial elongation factor G1 and combined oxidative phosphorylation deficiency. N Engl J Med. 2004;351(20): 2080-6. doi:10.1056/NEJMoa041878.

44. Smeitink JA, Elpeleg O, Zntonicka H, Diepstra H, Saada A, Smits $\mathrm{P}$, et al. Distinct clinical phenotypes associated with a mutation in the mitochondrial translation elongation factor EFTs. Am J Hum Genet. 2006;79(5):869-77. doi:10.1086/508434.

45. Desai SP, Bhatia SN, Toner M, Irimia D. Mitochondrial localization and the persistent migration of epithelial cancer cells. Biophys J. 2013;104(9):2077-88. doi:10.1016/j.bpj.2013.03.025.

46. Zhao J, Zhang J, Yu M, Xie Y, Huang Y, Wolff DW, et al. Mitochondrial dynamics regulates migration and invasion of breast cancer cells. Oncogene. 2013;32(40):4814-24. doi:10.1038/onc. 2012.494

47. Yu M, Shi Y, Wei X, Yang Y, Zang F, Niu R. Mitochondrial DNA depletion promotes impaired oxidative status and adaptive resistance to apoptosis in T47D breast cancer cells. Eur J Cancer Prev. 2009;18(6):445-57. doi:10.1097/CEJ.0b013e32832f9bd6.

48. Guha M, Avadhani NG. Mitochondrial retrograde signaling at the crossroads of tumor bioenergetics, genetics and epigenetics. Mitochondrion. 2013;13(6):577-91. doi:10.1016/j.mito.2013.08. 007.

49. Yu M. Generation, function and diagnostic value of mitochondrial DNA copy number alterations in human cancers. Life Sci. 2011;89(3-4):65-71. doi:10.1016/j.lfs.2011.05.010.

50. Cui H, Huang $\mathrm{P}$, Wang $\mathrm{Z}$, Zhang $\mathrm{Y}$, Zhang $\mathrm{Z}, \mathrm{Xu} \mathrm{W}$, et al. Association of decreased mitochondrial DNA content with the progression of colorectal cancer. BMC Cancer. 2013;13:110. doi:10. 1186/1471-2407-13-110.

51. Kirches E. Mitochondrial and nuclear genes of mitochondrial components in cancer. Curr Genom. 2009;10(4):281-93. doi:10.2174/ 138920209788488517. 
52. Elachouri G, Vidoni S, Zanna C, Pattyn A, Boukhaddaoui H, Gaget $\mathrm{K}$, et al. OPA1 links human mitochondrial genome maintenance to mtDNA replication and distribution. Genome Res. 2011;21(1):1220. doi:10.1101/gr.108696.
53. Krzywinski M, Schein J, Birol I, Connors J, Gascoyne R, Horsman D, et al. Circos: an information aesthetic for comparative genomics. Genome Res. 2009;19(9):1639-45. doi:10. 1101/gr.092759.109. 